\title{
PARIDAD DE GÉNERO Y REELECCIÓN: UN CONFLICTO ENTRE DERECHOS CIUDADANOS
}

\section{Octavio Grijalva Vásquez}

Sumario: I. Introducción. II. Preámbulo Histórico. III. Paridad de Género. IV. Reforma Político Electoral y maximización de los derechos políticos de las mujeres en su ámbito de aplicación. V. Reelección en Reforma 2014. VI. Reelección en Latinoamérica. VII. Reelección en México históricamente. VIII. Reelección en perspectiva del servidor público. IX. Reelección como derecho del ciudadano. X. Rendición de cuentas como prerrogativa ciudadana. XI. Profesionalización del poder legislativo y ediles. XII. Ponderación entre derechos constitucionales y valoración. XIII. Conclusiones.

\section{Resumen}

La democracia es una construcción jurídica y el derecho es el instrumento necesario para forjarla y garantizarla, según Bobbio. ${ }^{1}$

Para entender este garantismo, abordaremos la construcción normativa de nuestro país, específicamente de las figuras instauradas a partir de la reforma político-electoral de 2014, tales como la paridad de género y la reelección, utilizando la metodología de la dogmática jurídica, y el concepto de ponderación.

Esto permite realizar un esbozo de la situación venidera en la que ciudadanos y autoridades protagonizarán un interesante debate relacionado con la posible colisión entre derechos fundamentales y los nuevos postulados constitucionales, cuyo resultado no ha sido descifrado hasta la fecha.

Y a la vez proponer una solución a la problemática planteada entre ambos derechos, en el contexto actual en el cual se enfrentarán buscando cada una sobreponerse al otro, producto de un ejercicio ilegítimo de los ciudadanos.

Palabras Clave: Democracia, derechos fundamentales, paridad, relección.

\footnotetext{
* Actualmente Consejero Estatal Electoral de Sonora, Especialista en Derecho Penal y Criminología, Diplomado en el Nuevo Sistema de Justicia Penal Acusatorio y Oral por el INACIPE, Doctorando en Derecho por la Universidad de Baja California, Presidente del Colegio de Abogados del Estado de Sonora A.C.

${ }^{1}$ Norberto Bobbio, Introduziones aT. Hobbs, Opera Politiche (Turín: Utee, 1959), 23-24.
} 


\begin{abstract}
Democracy is a legal construction and law is the necessary instrument to build and enforce it, as per Bobbio.

In order to understand this guarantism we will discuss the regulating construction of our country, specifically in the figures established with the political-electoral reform as of 2014 , such as gender parity and reelection, using the legal dogmatics method and the concept of weighing.

This allows us to outline the upcoming situation where both citizens and authorities will play interesting roles in a debate linked to a potential collision between fundamental rights and the new constitutional postulates, which outcome has not yet been foreseen; at the same time proposing a solution to the issues between these two rights, in the present context where they will confront seeking to overcome one another, as a result of an electoral exercise by the citizens.
\end{abstract}

Key Words: Democracy, fundamental rights, parity, reelection.

\title{
Introducción
}

Hablar de democracia implica una valoración objetiva entre el derecho positivo y el bienestar social, partiendo del deber ser, para aterrizar en los factores reales del poder y asi lograr enderezar la ruta hacia su perfectibilidad. $^{2}$

Esta reflexión adquiere una connotación muy particular si partimos de la realidad de nuestro país, observando las garantías con que contamos actualmente hacia el mejoramiento de nuestras instituciones.

En este sentido, la Constitución federal como las leyes electorales se han robustecido mucho con las recientes reformas, que permiten su consolidación y fortalecimiento, especialmente, en cuanto a reelección y paridad de género se refiere, empero no se han aplicado totalmente.

\footnotetext{
${ }^{2}$ Ferdinand Lassalle, ¿Qué es una Constitución? ( Berlín: Ariel DERECHO, 2012), 47.
} 
De ahí que se considera que la situación que actualmente guarda nuestra democracia, se debe a la toma de importantes decisiones jurídicas, que como lo sugiere Jerzy Wróblewski ${ }^{3}$ son hasta el momento producto únicamente de quienes hacen el derecho.

Es decir, aún falta vislumbrar las variables para justificar las decisiones jurídicas que señala el autor, ya que están por verse los criterios interpretativos y la aplicación del derecho con las nuevas reglas del juego en el sistema político-electoral.

Como corolario, se tiene presente que se ha transitado de un régimen unipartidista, al multipartidismo y recientemente a gobiernos emanados de candidaturas independientes, y a las propuestas de gobiernos de coalición y de candidaturas comunes, lo que es y será muestra de la alternancia en muchos cargos de elección popular, algo casi impensable hace apenas 20 años.

Aunado a lo anterior, la construcción humanista de la Constitución Política de los Estados Unidos Mexicanos (Constitución Política), implica la exploración y la responsabilidad de estatuir a través de las nuevas codificaciones y la jurisprudencia, una sociedad que abiertamente privilegie ambos géneros en la participación política de manera igualitaria y plena.

De la misma forma el sistema político se encuentra a punto de implementar el antes criticado sistema de reelección consecutiva, que según la exposición de motivos del Decreto por el que se reforman, adicionan y derogan diversas disposiciones de la Constitución Política, en materia política-electoral, busca profesionalizar la función legislativa, incentivar la rendición de cuentas, mejorar la relación con el electorado, que permitan a la ciudadanía conservar y, a su vez, premiar las mejores formas de gobernar. ${ }^{4}$ Para elevar el principio de paridad y reelección a rango constitucional, se entiende que los Partidos Políticos convergieron en creencias, valores y aptitudes. No obstante, no se ha profundizado ante la posible colisión entre

\footnotetext{
3 Jerzy Wróblewski, Sentido y hecho en el derecho (México: Fontamara, 2008), 45.

${ }^{4}$ Decreto, por el que se reforman, adicionan y derogan diversas disposiciones de la Constitución Política, en materia política-electoral (Diario Oficial de la Federación, 10 de febrero de 2014), 51-53, http://www.dof.gob.mx/nota_detalle.php?codigo=5332025\&fecha=10/02/2014
} 
derechos fundamentales, materia de las recientes obligaciones y concesiones que se han hecho a las instituciones políticas, que a partir del próximo año, se observará de manera recurrente como producto del ideario de nuestra democracia.

En este sentido, destacaremos lo que estableció Max Weber citado por Pablo González Casanova, ${ }^{5}$ al señalar que la democracia tiene dos postulados fundamentales el primero, "impedir que se desarrolle un grupo cerrado de funcionarios oficiales para que exista posibilidad universal de acceder a los cargos públicos"; y el segundo, "la reducción de la autoridad para aumentar la esfera de influencia de la opinión pública".

De un análisis político de la historia de nuestro país, se advierte que el hecho de que tuviéramos un régimen autoritario dominado por un solo partido político, era considerado como algo negativo para nuestro sistema democrático, a ello habría de adicionar el análisis sociológico en el que se pone de relieve que el grupo cerrado de funcionarios referido en el párrafo anterior, también se encontró conformado casi en su totalidad por miembros del género masculino, excluyendo la posibilidad de participación de las mujeres en la política de nuestro país. ${ }^{6}$

\section{Preámbulo Histórico}

Al respecto, la teoría del género ${ }^{7}$ ubica el origen de esta desigualdad desde la ilustración, por el movimiento desarrollado en el siglo XVIII que derivó en el reconocimiento político-filosófico de atributos exclusivamente masculinos como la razón, la igualdad, la individualidad y la autonomía de los sujetos.

Esto significó que los reconocimientos de dichas cualidades se consideraban propias del género masculino, no así del género femenino.

\footnotetext{
${ }^{5}$ Pablo González Casanova, La Democracia en México (México: Era, 2008), 208.

${ }^{6}$ Ibíd., 110.

7 Yanko Molina Brizuela, "Teoría de Género en Contribuciones a las Ciencias Sociales", octubre 2010, http://www.eumed.net/rev/cccss/10/ymb2.htm
} 
Dicha circunstancia ha imperado como una patología política en casi todas las culturas del mundo, que el escenario internacional no ha tolerado y mucho menos permitido.

Ante ello, se han tomado las medidas para la protección de la igualdad de género en una amplia normatividad convencional de la cual nuestro país es parte.

Sin duda, todo esto ha tenido como finalidad proteger la igualdad de oportunidades y equidad entre los géneros, fomentando su participación en condiciones similares, y que ello pueda manifestarse de forma material o sustantiva en la composición de los órganos de toma de decisiones de los estados.

Por lo que, para lograr un estudio más apropiado es menester abordar los hechos más importantes alrededor del empoderamiento de la mujer; para ello, comenzaremos por mencionar el grado de limitación hacia las mujeres a nivel mundial, pues incluso durante el siglo XIX, se les impidió participar en la política, siendo hasta después de la segunda guerra mundial cuando la mayor parte de los países les otorgaron el derecho a votar.

Es en este contexto, de conformidad con los estudios realizados por la académica María Estela Báez-Villaseñor, ${ }^{8}$ surgió un primer movimiento internacional denominado sufraguismo, que respondió al monopolio del sufragio hasta ese momento dominado por el género masculino, lo que se extendió hasta la segunda mitad del siglo XX.

Fue hasta 1878 cuando se reunió el primer Congreso Internacional de Mujeres en Paris, teniendo una asistencia de 11 países. De esta manera, se fijó un piso que introdujo el sufragio femenino en Nueva Zelanda en 1893, Australia en 1901, así como Finlandia en 1906, quienes establecieron la pauta para que los demás países también reconocieran estos derechos.

En Estados Unidos de Norteámerica, el primer antecedente del sufragio femenino lo encontramos a nivel estatal a partir de 1869, en el estado en

\footnotetext{
${ }^{8}$ María Estela Báez-Villaseñor, Un largo camino: la lucha por el sufragio femenino en Estados Unidos ( México: Scielo, 2010), 16.
} 
Wyoming, pero fue hasta el término de la Primera Guerra Mundial en 1919 cuando se estatuye a nivel federal.

En América Latina, las naciones pioneras en incursionar en estos temas fueron Costa Rica, Brasil y Ecuador, y este último el primero en otorgar el sufragio femenino en 1929.

Ahora bien, al realizar un análisis de la evolución del voto femenino en el derecho comparado, tenemos un mal referente a nivel internacional, pues México, fue uno de los últimos en reformar su Constitución Política con el fin de incluir el sufragio femenino, haciéndolo hasta el año de 1953.

De igual manera, los principios de esta lucha de igualdad se encuentran en la Convención Sobre los Derechos Políticos de la Mujer, ${ }^{9}$ misma que, más allá del derecho del género femenino al sufragio, establece que tienen derecho a ser votadas para cargos de elección popular y puestos en la función pública, en igualdad de condiciones que los hombres.

Años más tarde, surgen las declaraciones, pactos, convenciones y conferencias, que protegen los derechos de la mujer, de manera que ésta podrá ejercer libre y plenamente sus derechos civiles, políticos, económicos, sociales y culturales, ya que, de no hacerlo, se estarían violentando sus derechos fundamentales.

Por otra parte, mientras todos estos cambios históricos, tenían lugar a nivel internacional, dentro de México, los primeros movimientos de mujeres se iniciaron en 1910, creando asociaciones con ideas feministas, que, unidas a Madero, protestaron por el fraude en las elecciones y demandaron la participación política de las mujeres mexicanas. ${ }^{10}$

Fue así que, en 1916, gracias al Primer Congreso Feminista apoyado por Salvador Alvarado entonces gobernador de Yucatán, se establecieron las bases para lograr el sufragio femenino.

\footnotetext{
${ }^{9}$ Convención sobre los derechos políticos de la Mujer (Nueva York: 31 de marzo de 1953), 2, disponible en: www.ordenjuridico.gob.mx/TratInt/Derechos\%20Humanos/D45.pdf

10 Esperanza Tuñón, "También somos protagonistas de la historia de México", Cuadernos para la Mujer (México: EMAS, 1987), 5-6.
} 
A pesar de esto, en la Constitución Política de 1917, no se declaró expresamente el derecho de la mujer al voto, siendo en 1937 cuando el Presidente Lázaro Cárdenas envió a la Cámara de Senadores la iniciativa para reformar el Artículo 34 constitucional, como primer paso para que las mujeres obtengan la ciudadanía, pero fue hasta 1946 cuando la Cámara de Diputados aprobó la iniciativa enviada por el Presidente Miguel Alemán, donde se adicionó al Artículo 115 de la Constitución Política, la facultad a las mujeres para participar en igualdad de condiciones que los varones, con el derecho a votar y ser elegidas, entrando en vigor el 12 de febrero de 1947, siendo así, como la mujer adquiere el derecho constitucionalmente a votar y ser votada, pero solo a nivel municipal. No obstante esto significaría un relevante antecedente de la reforma constitucional de 1953.

En efecto, el 17 de octubre de 1953, se hizo la publicación en el Diario Oficial de la Federación de la reforma al Artículo 34 de la Constitución Política, que otrogaría los mismos derechos a nivel federal, quedando de la siguiente manera:

"Son ciudadanos de la República los varones y las mujeres que, teniendo la calidad de mexicanos, reúnan, además, los siguientes requisitos: haber cumplido 18 años, siendo casados, o 21 si no lo son, y tener un modo honesto de vivir".

De lo anterior, se colige que la evolución del voto femenino en México descansó más de 40 años, lo que de suyo pone en evidencia una omisión ampliamente conocida en la que se les dejó de considerar para ocupar cargos de elección popular, quedando expuesta una profunda desigualdad.

Es ante estas circunstancias que surgen políticas de equidad, basadas en el reconocimiento de adoptar medidas como la discriminación positiva, que permitan luego de un lapso de tiempo, abatir y desterrar las diferencias entre mujeres y hombres.

En este sentido, la autora Alda Facio ${ }^{11}$ considera que las acciones afirmativas parten del reconocimiento de la histórica desigualdad de poder y goce de

\footnotetext{
${ }^{11}$ Alda Facio, El derecho a la igualdad de mujeres y hombres, Interpretación de los principios de igualdad y no discriminación para los derechos humanos de las mujeres en los instrumentos del Sistema Interamericano (San José, Costa Rica: IIDH, 2009), 73.
} 
derechos entre mujeres y hombres, obligando al Estado a tomar medidas especiales de carácter temporal para acelerar el logro de la igualdad.

Estas medidas también pueden comprenderse con un carácter correctivo, ante la herencia androcéntrica de carácter sistémica que ha estatuido la discriminación contra las mujeres, además de la evidente falta de una masa crítica de mujeres en puestos de autoridad y de toma de decisiones, por lo que el reconocimiento de estas diferencias implica acciones inmediatas traducidas en reglas que beneficien concretamente a las mujeres.

En este punto, es necesario hablar del antecedente más inmediato en nuestro país al respecto, las cuotas de género, que surgen como una medida para encontrar el equilibrio entre la participación de hombres y mujeres en los órganos de toma de decisiones. ${ }^{12}$

Así, en 1996, estas cuotas se establecieron para ser compuestas por un setenta por ciento de legisladores de un género y un treinta por ciento del otro, otorgando siempre mayoría a los hombres, creándose así el sistema de listas de cremallera para evitar que se relegara a las mujeres a los peores sitios electorales. $^{13}$

Las listas cremalleras se integraban por segmentos de tres candidaturas, dónde en cada uno de los tres primeros segmentos por lista, había una candidatura de género distinto.

Estas medidas en términos coloquiales se pueden entender como la previsión normativa de un umbral mínimo de mujeres que debían ser propuestas como candidatas, cuyo objeto era lograr el ejercicio pleno de la ciudadanía.

Este umbral ha avanzado eliminando la brecha de desigualdad entre mujeres y hombres, y es en este sentido donde en el camino de las reformas ha aparecido la paridad.

Y fue en la reforma de 2008 , donde se estableció como tal el término de "paridad de género" en lugar de "equidad entre hombres y mujeres". De igual

\footnotetext{
12 Ibíd., 74.

${ }^{13}$ Lisa Baldez, "Cuotas versus primarias: La nominación de candidaturas mujeres en México", En Mujer y Política: Impacto de las cuotas de género en Améerica Latina (Chile: Catalonia, 2012), 15777.
} 
manera, cambiaron los porcentajes de las formulas, integrándose con al menos cuarenta por ciento de candidatos propietarios de un mismo género, impulsando con ello la participación de la mujer, mediante el otorgamiento de diez por ciento más candidaturas al género menos favorecido.

Dicha circunstancia si bien representó un nuevo adelanto cuantitativo hacia el respeto y reconocimiento del valor y capacidad de la mujer para ocupar cargos públicos, resultó insuficiente ante la eventual reforma histórica en materia de derechos humanos que se dio en el año 2011.

Después de lo anterior, surge la paridad de género como la conocemos en la actualidad.

\section{Paridad de Género}

La paridad de género ha resultado realmente complicada, debido a la arraigada cultura del patriarcado y "androcentrismo" (visión del mundo y de las relaciones sociales centrada en el punto de vista masculino) ${ }^{14}$, que aún existe en nuestra sociedad.

Por ello, es necesario hacer referencia a ciertos sucesos históricos que son indispensables para lograr entender el fenómeno que se aborda.

El 10 de febrero de 2014, se publicó en el Diario Oficial de la Federación, el decreto por el que se reforman, adicionan y derogan diversas disposiciones de la Constitución Política, en materia política-electoral, mediante el cual, en su artículo 41, fracción I, segundo párrafo, se dispuso entre otras cosas, lo siguiente;

"Los partidos políticos tienen como fin promover la participación del pueblo en la vida democrática, contribuir a la integración de los órganos de representación política y como organizaciones de ciudadanos, hacer posible el acceso de éstos al ejercicio del poder público, de acuerdo con los programas, principios e ideas que postulan y mediante el sufragio universal, libre, secreto y directo, así como las reglas para garantizar la paridad entre los géneros, en candidaturas a legisladores federales y locales".

\footnotetext{
${ }^{14}$ RAE, Diccionario de la Lengua Española, 23 va ed., s. v. “androcentrismo”, Madrid, 2014.
} 
Derivado de la anterior reforma, con fecha de 23 de mayo de 2014, se publicó en el Diario Oficial de la Federación el Decreto por el que se expiden la Ley General de Instituciones y Procedimientos Electorales (LEGIPE) y la Ley General de Partidos Políticos (LGPP), las cuales establecen nuevas disposiciones en la participación de las mujeres en la vida política, para ser electas en los cargos de representación popular.

Con la anterior reforma, surge como obligación de los partidos políticos promover la paridad de género en candidaturas destinando el $3 \%$ de su gasto ordinario para capacitar a mujeres, así mismo, ha facultado al Consejo General del Instituto Nacional Electoral (INE), para rechazar el registro de las candidaturas que no respeten el principio de paridad de género.

Este antecedente inmerso en un cúmulo de modificaciones políticas en nuestro país, impactó en la evolución de la paridad de género, estableciendo un sistema tendente a reafirmar los derechos político-electorales de las mujeres, que como a continuación veremos, cuenta con algunos puntos inexplorados.

También tuvo un impacto en los mencionados ordenamientos con el propósito de alcanzar una efectiva paridad de género, pués se establecen varios principios, tales como las fórmulas para senadurías y diputaciones por los principios de mayoría relativa y representación proporcional, que deberán integrarse por personas del mismo género, al igual que con las candidaturas independientes, así como la no asignación exclusiva a un género en distritos de baja votación.

De igual forma, encontramos que es responsabilidad de los partidos políticos promover y garantizar la paridad de género, en la participación de mujeres y hombres en condición de igualdad, respetando las normas constitucionales federales y locales, así como las leyes en materia estatal aplicables.

Y se estipuló que las fórmulas de candidatos, tanto por mayoría relativa como por representación proporcional, deberán ser compuestas con personas del mismo género, así como la totalidad de solicitudes de registro de las candidaturas a diputados y senadores que presenten los partidos políticos o coaliciones, deberán integrarse salvaguardando la paridad entre 
los géneros, y en las listas de representación proporcional se alternarán las fórmulas de distinto género para garantizar el principio de paridad hasta agotar cada lista.

En adición a lo anterior, se tiene el criterio sustentado por la Sala Superior del Tribunal Electoral del Poder Judicial de la Federación (Sala Superior), mediante la jurisprudencia $6 / 2015,{ }^{15}$ en el sentido de que el principio de paridad:

"emerge como un parámetro de validez que dimana del mandato constitucional y convencional de establecer normas para garantizar el registro de candidaturas acordes con tal principio, así como medidas de todo tipo para su efectivo cumplimiento, por lo que debe permear en la postulación de candidaturas para la integración de los órganos de representación popular tanto federales, locales como municipales, a efecto de garantizar un modelo plural e incluyente de participación política en los distintos ámbitos de gobierno".

Todo lo anterior, permite establecer metas novedosas para los partidos políticos en México, pasando de un discurso oficial a un ámbito operativo como lo explica Gema Sánchez Medero, ${ }^{16}$ al señalar que las organizaciones políticas no solo tienen un objetivo (a diferencia del concepto establecido en la Constitución Política), pues tienen diferentes objetivos que van cambiando para adaptarse a su entorno y de esta manera, conseguir su objetivo principal.

Así, para este nuevo episodio de nuestra democracia, motivado en la reforma que a continuación se revisa, se observa que el discurso concebido originalmente, como un matiz general desde el ámbito constitucional al hablar de promover la participación del pueblo en la vida democrática del país, viene a convertirse a una afirmación más específica que incluye a

\footnotetext{
${ }^{15}$ PARIDAD DE GÉNERO. DEBE OBSERVARSE EN LA POSTULACIÓN DE CANDIDATURAS PARA LA INTEGRACIÓN DE ÓRGANOS DE REPRESENTACIÓN POPULAR FEDERALES, ESTATALES Y MUNICIPALES, 6/2015 ( 6 de mayo de 2015, Gaceta de Jurisprudencia y Tesis en materia electoral, Tribunal Electoral del Poder Judicial de la Federación, Año 8, Número 16, 2015), 24- 26.

${ }^{16}$ Gema Sánchez Medero, Los Partidos Políticos: Organización y Funcionamiento (Madrid: Trotta, 2012), 141.
} 
ambos géneros en la mencionada promoción y a formar parte de la propia finalidad de los partidos políticos.

\section{Reforma Político Electoral y maximización de los derechos políticos de las mujeres en su ámbito de aplicación}

Por medio de esta reforma realizada en 2014 , se incorporó por primera vez en nuestro país, el deber de los partidos políticos de buscar la participación efectiva de ambos géneros en la postulación de candidaturas, por lo que el INE, desarrolló para su primer acto de aplicación, una serie de acciones con el propósito de lograr el registro paritario de candidaturas.

Tal como sucedió en el proceso electoral del año 2015, donde Sala Superior al resolver el Recurso de Apelación SUP-RAP-134/2015, ${ }^{17}$ confirmó el acuerdo INE/CG162/2015, mediante el cual, se determinó la aplicación de criterios basados en bloques de mayor, mediana y menor votación por partido político en la asignación de candidaturas.

Ello, con la finalidad de que en la postulación a cargos de elección popular se garantizara la efectiva participación política de ambos géneros sin que se otorgaran las candidaturas con menor probabilidad de triunfo a un determinado grupo, considerando la votación que recibió cada partido político en la elección anterior, es decir, que la paridad no sea únicamente cumplir con la mitad de las postulaciones de un mismo género, sino que también se evitara que un género obtuviera las posiciones menos competitivas.

Dicho de otro modo, se buscó generar una proporcionalidad de oportunidades para obtener la mayoría de sufragios, sin que existieran sesgos evidentes de candidaturas en distritos históricamente desfavorables para algún partido.

Esta nueva conformación se dio a raíz de que un partido político impugnó, entre otras cosas, la aplicación de dicho criterio, argumentando que la autoridad excedió lo establecido en la legislación atinente, y razonó que la

\footnotetext{
17 Sentencia SUP-RAP-134/2015, (Tribunal Electoral del Poder Judicial de la Federación de 6 de mayo de 2015).
} 
autoridad electoral estaba regulando de manera excesiva e incluso legislando aun cuando carecía de atribuciones para tales efectos, empero, Sala Superior razonó que no se trató de una intromisión excesiva de la autoridad, en virtud de que el INE generó el método idóneo para observar lo contenido en el artículo 3 párrafo 5 de la LGPP, en relación a que no se admitirán criterios que posibiliten que alguno de los géneros le sean asignados exclusivamente los distritos en los que el partido político haya obtenido la votación más baja en el proceso electoral anterior, considerando que se trata de una acción afirmativa en favor del género históricamente relegado.

Criterio que la Sala Superior ha reiterado recientemente mediante SUPREC-825/2016, ${ }^{18}$ donde deja en claro varias aspectos controvertidos, tales como son el interés jurídico para interponer los medios de impugnación en su calidad de mujeres pertenecientes a un grupo vulnerable, la maximización del derecho pleno de acceso a la justicia, la no vulneración del principio de libertad configurativa legislativa, la facultad reglamentaria de los Organismos Públicos Locales (OPLES) para la emisión de lineamientos, la no afectación a los derechos de auto-organización y autodeterminación de los partidos, al emitir lineamientos con acciones afirmativas de género.

De esta manera, se ha avanzado en la igualdad entre géneros en la participación política, aunado a otras determinaciones que el máximo órgano jurisdiccional electoral del país ha emitido en la materia.

De todo lo anterior, podemos concluir válidamente que con la reforma político electoral de 2014 , los criterios y lineamientos del INE, la jurisprudencia pro derechos humanos del Tribunal Electoral del Poder Judicial de la Federación, haciendo una interpretación conforme a la Constitución Política y los tratados internacionales, México pone la pauta como una de las naciones que buscan acelerar el ritmo para lograr la igualdad sustantiva, esto es la igualdad material entre los géneros, es decir,

\footnotetext{
18 Sentencia SUP-RAP-825/2016, ( Tribunal Electoral del Poder Judicial de la Federación, de 21 de diciembre de 2016).
} 
ya no solo el acto mismo de la postulación y registro, sino también en el hecho de poder acceder al cargo. ${ }^{19}$

Pero no obstante esa tendencia de las autoridades electorales a cerrar la brecha generacional de desigualdad entre mujeres y hombres, se avecina en las próximas elecciones de 2018, el ejercicio inédito de la figura de la reelección, que buscará anteponerse a la paridad de género, como un derechos ciudadano o prerrogativa política del gobernante en funciones a reelegirse y de los propios ciudadanos a reelegirlo. De ahí que resulte necesario abordar ampliamente la reelección, no solo a partir de la reciente reforma político-electoral, sino a través de la historia tanto de México como de los países de Latinoamérica, como seguidamente se hará.

\section{Reelección en Reforma 2014}

En el presente artículo, se ha abordar el estudio de diferentes reformas electorales a lo largo de la historia, para comprender qué es la paridad de género en este ámbito y cómo ha evolucionado nuestro marco legal, para una participación equitativa entre mujeres y hombres.

Como se mencionó, por medio de la reforma del 10 de febrero de 2014, se realizaron cambios importantes para lograr mayor participación de la mujer dentro de sus partidos políticos. Paralelamente, tuvieron lugar otros cambios de relevancia histórica, como la figura de la reelección.

De esta manera, la ciudadanía será capaz de decidir si reeligen o no a los diputados federales y senadores que hubieren sido electos a partir del proceso electoral de 2018, cuando sean postulados de nueva cuenta para el mismo cargo.

Por otra parte, se estableció que la reelección consecutiva de legisladores de las entidades federativas y ediles de los ayuntamientos, dependerá de las reformas que se realicen a las constituciones locales.

\footnotetext{
${ }^{19}$ ACCIONES AFIRMATIVAS. TIENEN SUSTENTO EN EL PRINCIPIO CONSTITUCIONAL Y CONVENCIONAL DE IGUALDAD MATERIAL, Jurisprudencia 43/2014 (29 de octubre de 2014, Gaceta de Jurisprudencia y Tesis en materia electoral, Tribunal Electoral del Poder Judicial de la Federación, Año 7, Número 15), 12-13.
} 
En ese sentido, bajo una interpretación lógica de los transitorios Décimo Tercero y Décimo Cuarto de la reforma, la reelección de diputados locales, Asamblea Legislativa del otrora Distrito Federal hoy Ciudad de México, presidentes municipales, regidores y síndicos, al establecer que no será aplicable a los que se encontraban en funciones a la entrada en vigor del decreto, podría ser válido concluir que los electos en procesos electorales 2014-2015, tendrán acceso a la relección.

En el ámbito federal ésta figura consiste en que, los senadores podrán reelegirse en dos períodos consecutivos, mientras que los diputados del Congreso de la Unión, hasta por cuatro períodos consecutivos, de esta forma las autoridades legislativas podrían permanecer en su cargo hasta por doce años. ${ }^{20}$

En cambio, en el ámbito local se ordenó realizar las correspondientes reformas en las respectivas constituciones políticas para prever la figura de la reelección consecutiva de diputados hasta por cuatro períodos y a ediles por un período adicional, siempre y cuando el período del mandato del Ayuntamiento no sea superior a tres años. ${ }^{21}$

También en la citada reforma, se establece el procedimiento por el que deberá realizarse, que en todos los casos tendrá que ser por el mismo partido político o coalición que los postuló, o por la vía de una candidatura independiente, con la excepción de que éste haya renunciado o perdido su militancia antes de la mitad de su mandato.

Entre las ventajas de esta reforma, se prevé que los ciudadanos logren un vínculo más estrecho con sus representantes, pues servirá como un medio de ratificación de su labor, además de la rendición de cuentas, la profesionalización del ejercicio parlamentario y la continuidad de las decisiones que ofrezcan mejores resultados. ${ }^{22}$

\footnotetext{
${ }^{20}$ Constitución Política de los Estados Unidos Mexicanos (México: Instituto Nacional Electoral, Fiscalía Especializada para la Atención de Delitos Electorales, Instituto de Investigaciones Jurídicas de la Universidad Nacional Autónoma de México y Tribunal Electoral del Poder Judicial de la Federación, 2014), artículo 59.

${ }^{21}$ Ibíd., artículos 115 y 16 .

${ }^{22}$ Decreto, Diario Oficial de la Federación, (véase, n 5 ), 1-2.
} 


\section{Reelección en Latinoamérica}

En América Latina existen diversos ejemplos históricos de la tergiversación de la reelección que se han convertido en dictaduras omnipotentes e inamovibles, con las eventuales insurrecciones sociales ocasionadas por la perpetuación de gobernantes por encima de la voluntad popular en detrimento de sus derechos y el bienestar de la colectividad, que es el fin mismo del Estado, el cual da origen al sistema democrático para la selección de los representantes populares. ${ }^{23}$

En ese sentido, la tendencia actual se encuentra dirigida a la aceptación de la figura de la reelección mediante el fortalecimiento de los sistemas democráticos, imponiendo la obligación de no reconocer gobiernos que no sean emanados de procesos ciudadanizados, en concordancia, la propia Carta Democrática Interamericana, ${ }^{24}$ establece como obligación de todos los estados que integran la Organización de Estados Americanos, promover y defender dichos valores.

Esto es, se reconoce la evolución de las democracias americanas, como garantía de que los sistemas actuales contendrán elementos con los que se asegure la no repetición de regímenes dictatoriales, resultado de procesos antidemocráticos que atenten contra el derecho colectivo de elegir libremente a sus gobernantes, incluso como se plasma en la citada Carta, bajo la condición de no reconocer gobiernos emanados en contravención a ello.

\section{Reelección en México históricamente}

En el contexto mexicano del siglo $X X$, se ha estigmatizado la figura de la reelección de los servidores públicos electos popularmente, es decir, por

\footnotetext{
${ }^{23}$ Carlos Alfonso Calderon Hernández, "La reelección presidencial en latinoamerciana: una avance en el caudillismo y un retroceso en la democracia”, Derechos y Realidad, n, 24, Il sementre (2014): 12830.

${ }^{24}$ Carta Democrática Interamericana, OEA, (Lima Perú: 11 de septiembre de 2001), disponible en: www.ordenjuridico.gob.mx/TratInt/Derechos\%20Humanos/OTROS\%2000.pdf
} 
medio de la expresión ciudadana ejercida a través de elecciones democráticas. ${ }^{25}$

El antecedente más significativo de la voluntad ciudadana por la que se determinó la prohibición a la reelección, se dio durante el "Porfiriato" a inicios del siglo $\mathrm{XX}$ exclusivamente para el cargo de Presidente, no así para los cargos legislativos, que tuvieron vigencia hasta la reforma de $1933,{ }^{26}$ en la que también fueron excluidos de la reelección.

En la reforma político-electoral del 2014, se incluyó la figura de la reelección para Senadores, Diputados y Ediles, a través de la modificación de los artículos 59, 115 y 116 de la Constitución Política, ${ }^{27}$ así como los transitorios Décimo Tercero y Décimo Cuarto de la misma.

Las consideraciones principales expuestas en el Congreso de la Unión para retirar la prohibición de la reelección, las podemos señalar en tres ejes torales: 28

1. Altos costos, para la calidad de gobierno que se tiene y su poca relación entre ciudadanos y representantes electos.

2. Promover la profesionalización de los representantes legislativos y autoridades municipales, generando el aprovechamiento de la experiencia en el cargo respectivo.

3. Permitir a los ciudadanos aprobar o no la gestión de sus representantes en el desempeño del encargo.

De los puntos anteriores, se puede observar que en gran medida, se trata de intereses colectivos tanto económicos como sociales respecto al costo de la elección de los representantes populares, así como de la necesidad social

\footnotetext{
${ }^{25}$ Dieter Nohlen, "La reelección", cap. XV en Tratado de Derecho Electoral Comparado de América Latina (México: Fondo de Cultura Económica, Instituto Interamericano de Derechos Humanos, Universidad de Heidelberg, International IDEA, Tribunal Electoral del Poder Judicial de la Federación, Instituto Federal Electoral, 2007).

${ }^{26}$ Ibíd., 286-86.

${ }^{27}$ CPEUM., Artículos 59, 115, 116, Transitorios Décimo Tercero y Décimo Cuarto.

${ }^{28}$ Iniciativa presentada por la Senadora Mariana Gómez del Campo Guza, con proyecto de decreto por el que se reforman los artículos 59, el segundo párrafo de la fracción II del artículo 116 y segundo párrafo de la fracción I base primera del artículo 122 de la Constitución Politíca del los Estados Unidos Mexicanos (Camara de Senadores, 9 de octubre de 2012, públicada en el Diario Oficial de la Federación el 10 de febrero de 2014, como Decreto por el que se reforman, adicionan y derogan diversas disposiciones de la Constitución Política de los Estados Unidos Mexicanos, en materia político electoral), 49-50.
} 
de estar en condiciones de realizar un escrutinio sobre el desempeño de sus representantes, para determinar sobre su idoneidad y estar en aptitud de definir sobre la posibilidad de un nuevo período consecutivo en el encargo. En ese sentido, es necesario destacar que dicho interés colectivo no debería encontrar distingo alguno, toda vez que en México contamos con un sistema democrático de elección directa mediante el sufragio de la ciudadanía. ${ }^{29}$ De ahí que, implique un derecho inherente a todas las personas, de reelegir a sus representantes populares, el cual no debe ser restringido por algún, como podría entenderse en este caso el de paridad sustentiva en materia electoral, cuyas acciones afirmativas en favor de las mujeres podrían contraponerse con un derecho que compete a todas las personas, no solo a un grupo por más numeroso y relevante que este sea, lo cual ineludiblemente ocasionaría la vulneración de los principios de interdependencia y de indivisibilidad de los derechos fundamentales. ${ }^{30}$

\section{Reelección en perspectiva del servidor público}

Los servidores públicos electos mediante el voto directo del electorado, al momento de ostentar el cargo público, lo hacen como consecuencia de haber contendido en una campaña electoral, con todas sus implicaciones, desde reunir los requisitos legales indispensables hasta la consecución de la confianza ciudadana reflejada en las urnas, incluso en la mayoría de los casos, después de enfrentar batallas en los tribunales, que en la época moderna se han convertido en la última instancia para la convalidación de los resultados electorales.

Considerando lo anterior, es evidente que obtener cargos de elección popular requiere de una cantidad importante de recursos que primordialmente tienen un origen público. ${ }^{31}$

En el caso particular de los servidores electos democráticamente, en la historia reciente se les ha impedido reelegirse, sin que exista vía alguna para

\footnotetext{
${ }^{29}$ CPEUM., Artículo 34.

30 PRINCIPIOS DE UNIVERSALIDAD, INTERDEPENDENCIA, INDIVISIBILIDAD Y PROGRESIVIDAD DE LOS DERECHOS HUMANOS. EN QUÉ CONSISTEN, Tesis 2003350 I.4o.A.9 K (10a) (Abril de 2013, Libro XIX, Tomo 3, Semanario Judicial de la Federación y su Gaceta), 2254.

${ }^{31}$ CPEUM., Artículo 41 fracción II.
} 
tener la oportunidad de continuar en el cargo, con independencia del desempeño satisfactorio o negativo que hayan realizado.

En tales circunstancias, la incorporación de la figura de la reelección consecutiva de legisladores y ediles, como se establece en la exposición de motivo de la citada reforma, expande el margen de acción de los funcionarios para realizar gestiones efectivas encauzadas a "conectar con el sector de la sociedad" al que representan, que si bien es cierto, tratándose de servicio público en todo momento y circunstancia idealmente deberían observase, también lo es que sin la figura de la reelección, el ostentante del cargo puede no tener "incentivos" para ejercer de manera óptima sus funciones, por ende, encontrarse ante la oportunidad de que la empatía que genere con sus electores sea materializada mediante la reelección en el cargo, equivale a una maximización de su derecho a participar en la vida política, así como la evaluación directa de quienes primigeniamente ostentan la residencia de la soberanía, es decir, del pueblo. ${ }^{32}$

Se concluye, que la reelección para los servidores públicos con independencia de su género, es un derecho que como ciudadanos les corresponde en términos de la Constitución Política, por lo tanto, deben tutelarse de manera irrestricta en correlación con otros derechos tutelados constitucionalmente, como el de paridad entre los géneros, atendiendo invariablemente a los principios de interdependencia e indivisibilidad de los derechos humanos.

\section{Reelección como derecho del ciudadano}

La figura de la reelección no debe ser entendida exclusivamente como una prerrogativa en favor de quien detenta un cargo público de elección popular, por el contrario, es un efecto colateral al interés superior de la colectividad sobre el personal de cualquier Legislador o Edil. ${ }^{33}$

\footnotetext{
${ }^{32}$ Decreto, Diario Oficial de la Federación, (véase, n. 5), 49-50.

33 Enrico Spagna Musso, "La Tutela Constitucional de los intereses colectivos en el Estado de Democracia Pluralista", Estudios Políticos del Centro de Estudios Constitucionales de Madrid, n. 4 (1978): 59.
} 
En una sociedad, máxime una de carácter democrático, el mayor interés que se debe tutelar por el Estado, es el de la totalidad de los miembros que la conforman, por encima de cualquier interés de grupos o personas ${ }^{34}$, en ese tenor, es importante reflexionar sobre la reelección como derecho de los ciudadanos, más allá de las implicaciones hacia los candidatos o servidores públicos.

De conformidad con la Constitución Política y los tratados internacionales como la propia Declaración Universal de los Derechos Humanos o el Pacto Internacional de los Derechos Civiles y Políticos, los ciudadanos tienen el derecho de votar y ser votados en elecciones periódicas, auténticas, entre otras características.

Lo cual se extiende al derecho que ostenta la población de participar en la vida política en la vertiente de elegir libremente a sus representantes legislativos y edilicios, sin restricciones legales más allá de los requisitos de elegibilidad que cada encargo requiere por su propia naturaleza.

Tal derecho no debe ser restringido de manera alguna, toda vez que la opción ciudadana de elegir nuevamente a una persona que ha ostentado un cargo público, le permite generar un juicio de valor sobre la actuación de su representante, su conocimiento e idoneidad para ejercer la función pública, así como reconocerla o desaprobarla, de lo anterior es posible diseccionar diversas cuestiones inherentes a la voluntad de la ciudadanía en su conjunto.

En primer término, la sociedad tiene interés en el desempeño de sus gobernantes en función del bienestar que estos generen, esto es, observar los resultados tangibles -positivos o negativos- de las gestiones realizadas por sus autoridades, con relativa independencia de cuestiones subjetivas, sino lo que la sociedad percibe que sucede en su entorno, por lo tanto, el primer motivo de escrutinio de la ciudadanía radica en lo que consideran ha generado la actuación del funcionario del que se trate.

Mediante esa valoración, la colectividad quienes ostentan el máximo derecho para determinar la continuidad en el cargo del gobernante, contarán 
con elementos para dilucidar al respecto, toda vez que uno de los factores de su elección es el desempeño en la gestión, de lo contrario se está en aptitud de buscar la alternancia.

Siguiendo la lógica de la alternancia, podemos considerar que la reelección es de similar relevancia para la consecución de la voluntad ciudadana de tener a los gobernantes considerados aptos por los electores, puesto que si es su voluntad el cambiar a las personas que los representan cuando no están de acuerdo en su gestión, resulta lógico considerar que debe existir la oportunidad de darle continuidad a una gestión aprobada.

Así mismo, en intrínseca relación con lo señalado, la sociedad que elige a sus gobernantes de forma democrática, cuenta con el derecho para determinar si con base en el desempeño de su encargo y la experiencia que pudieren haber adquirido en el ejercicio de sus funciones, deban ser reelectos, independientemente del género del gobernante.

Por ello, es menester que a la sociedad se le permita estar en condiciones de elegir a quien por su desempeño y aptitud consideren cuente con la experiencia necesaria para repetir en su encargo, en caso de así considerarlo.

\section{Rendición de cuentas como prerrogativa ciudadana}

En el contexto actual, la rendición de cuentas de los servidores públicos, es un factor indispensable para el escrutinio de las gestiones realizadas por los gobernantes.

En ese sentido, es imprescindible potencializar la explicación de determinaciones y gestiones de los gobernantes ante sus electores, como un método en el que los funcionarios realmente se sitúen compelidos a ejercer con probidad los cargos públicos, no sólo por el temor de ser objeto de medidas coercitivas, sino además por la posibilidad de incidir bajo esa transparencia en su actuar, para buscar la reelección en su encargo.

Mediante la rendición de cuentas, se creará la posibilidad de que el ciudadano pueda ejercer con mayor plenitud sus derechos por medio del sufragio directo, puesto que la percepción que el votante tenga en relación a 
su Legislador o Edil obtenida mediante el exhaustivo escrutinio de las funciones desempeñadas, le permitirá ejercer su derecho con una criterio mayormente informado.

Así las cosas, la generación de mecanismos dirigidos a la explanación de la actuación de los servidores públicos ante la ciudadanía, resultan de la mayor importancia en la obtención de una verdadera profesionalización del servicio público, ${ }^{35}$ así como del ejercicio de los derechos político-electorales de la colectividad mediante el sufragio activo.

De nueva cuenta, tenemos que se trata de la optimización de los órganos de gobierno, así como de los derechos de una colectividad compuesta por ambos géneros en la búsqueda del bienestar de la comunidad, por tal motivo es que se debe reflexionar severamente acerca de la pertinencia o no de soslayar lo anterior por la aplicación de acciones afirmativas de género de carácter temporal, en favor de un porcentaje mayoritario de la sociedad.

\section{Profesionalización del poder legislativo y ediles}

En cualquier rama del conocimiento humano, el discernimiento de las cuestiones inherentes a la materia de que se trate, el descubrimiento de información, desarrollo del conocimiento y comprensión del objeto de estudio, se genera mediante la práctica en determinado ambiente 0 circunstancia para allegar un mayor conocimiento de la materia en estudio. ${ }^{36}$ El campo del ejercicio de los puestos legislativos y edilicios, no es ajeno a esa circunstancia, puesto que un mayor conocimiento de las actividades que deben realizar con motivo de las funciones ejercidas, otorga mayor grado de ilustración en relación con los deberes propios de la función pública correspondiente.

Puntualizando que no se afirma categóricamente que quien haya ostentado un cargo, forzosamente tendrá mejores condiciones de las que cualquier ciudadano que no ha fungido en el encargo, puesto que no es

\footnotetext{
${ }^{35}$ David Jaimes Delgado, La Reelección Legislativa inmediata una propuesta para fortalecer el Poder Legislativo en México (México: Porrúa, 2011), 127.

${ }^{36}$ Raúl Gutiérrez S., Los elementos del conocimiento en general (México: Esfinge, 2000), 18-19.
} 
exclusivamente mediante el ejercicio del cargo que se puede obtener sapiencia al respecto.

Tampoco se afirmar que la reelección sea la forma como se debe elegir a los gobernantes únicamente, pues hacerlo es caer en el absurdo de perpetuar a los funcionarios, bajo el argumento de la experiencia adquirida, no obstante, tampoco debe soslayarse la relevancia de lo aprendido durante el ejercicio del encargo público.

\section{Ponderación entre derechos constitucionales y valoración}

Con la proximidad de las elecciones a celebrarse en el año 2018, nos encontraremos ante el escenario en el cual se dé por primera vez la confrontación entre la paridad de género y la reelección consecutiva.

Este nuevo horizonte pondrá a prueba nuestras instituciones tanto jurisdiccionales como administrativas, pues sin duda la posibilidad de su ponderación para decidir cuál debe prevalecer, generará un debate interesante entre los derechos de un género contra los de la colectividad en la que se encuentran incluidos ambos género.

Producto de los debates en las instancias mencionadas, observaremos la solidez y consolidación de nuestra democracia, en la construcción de decisiones jurídicas que van más allá del plano legislativo, que terminarán de dibujar el ideario Constitucional.

En ese sentido, la paridad de género ha adquirido un estatus relevante en función de su previsión constitucional, legal, así como a través de acciones afirmativas en acuerdos administrativos y sentencias judiciales, además tienen un arraigo jurisprudencial que cada día se está acrecentando, lo que de cierta manera, ha dejado un camino tendente a la consolidación sustantiva de este principio.

No obstante, el desfase que representa su adelantada implementación de la paridad en comparación con el derecho a la reelección consecutiva, que se estatuyo como una garantía tanto para el ciudadano que desempeñe el cargo como para la propia colectividad, esto no significa supremacía de un derechos sobre le otro. 
Es cierto, a la fecha no se encuentra regulada la aplicación de la disposición constitucional relativa a la reelección, que como se ha sostenido, es de interés general, e impacta de manera idéntica a ambos géneros dando la posibilidad al género femenino de obtener de nueva cuenta el cargo alcanzado mediante las elecciones en el proceso electoral previo.

Sin embargo, atendiendo a los principios de indivisibilidad e interdependencia de los derechos fundamentales, es que debemos ponderar la relevancia que tienen en correlación la paridad de género y la reelección consecutiva, puesto que ambas son detonantes para la maximización de los derechos y una democracia efectiva alejada de obcecaciones de antaño.

Finalmente, es importante señalar que el derecho a la reelección consecutiva encuentra un trasfondo aún más profundo y significativo, que el establecer un método para premiar a los gobernantes, como lo son la transparencia, rendición de cuentas, profesionalización en el desempeño del servicio público y empoderamiento del electorado frente a sus representantes.

En el plano jurídico y filosófico, podemos considerar que a la postre, la progresividad y la indivisibilidad que son características de los derechos humanos, generarán un amplio conflicto que se desahogará sin duda en tribunales.

\section{Conclusiones}

- Como se desprende de la norma constitucional, resulta evidente que tanto la reelección y la paridad de género, son elementos inalienables de derechos de los ciudadanos para ejercer cargos públicos de elección popular, los cuales no tienen jerarquía entre ellos, es decir, son interdependientes.

- Para dilucidar acerca del posible conflicto expuesto, es menester atender a la finalidad y cercanía del objetivo de cada uno de los derechos, así como su viabilidad en el campo de la práctica, esto es, la consecución de los fines.

- Se ha reseñado que la paridad de género contiene avances significativos a través de acciones legislativas y judiciales, maximizando los derechos del 
grupo históricamente afectado por la ausencia de medios para garantizar el cumplimiento de dicho principio, avances que en la actualidad han contribuido a la igualdad entre géneros para la postulación de candidatos, tendente a lograrla también en el ejercicio de los cargos públicos, contribuyendo a la materialización objetiva de los derechos de los destinatarios del imperativo legal, es decir, de todas las ciudadanas, y en general, se la colectividad.

- Por el contrario, si bien es cierto que por medio de la reforma electoral 2014, se implementó la reelección consecutiva de legisladores y ediles, aun cuando teleológicamente este es un derecho más amplio por tratarse de derechos que incluyen a hombres y mujeres como destinatarios, también lo es que no se trata de un ejercicio que sea específicamente dirigido a la ciudadanía, esto es así en virtud de que la forma en la que se encuentra legislado, constriñe y condiciona el ejercicio de ese derecho, a la potestad de un partido político el someter a reelección a un funcionario público, apartándolo de ser un tema ciudadano.

- Dicho de otra manera, los ciudadanos no ostentan poder de decisión para postular a quien se vaya a reelegir, siendo que en el derecho a la paridad, si se estará en presencia de una prerrogativa ciudadana para determinar sobre la elección de sus representantes populares.

- Por lo tanto, al estar la reelección supeditada a las decisiones de partidos políticos, y no de la ciudadanía, es que para el próximo proceso electoral deberá continuarse por la senda de la consecución efectiva de la paridad de género, privilegiándose -en caso de controversia con la reelección consecutiva de legisladores y ediles- la paridad de género, por ser ésta una causa afin a los intereses y derechos de los ciudadanos, por encima como el de entidades como los partidos políticos.

- Sin perjuicio, de que deberán crearse los mecanismos necesarios para que en el futuro la reelección pueda realizarse como un derecho totalmente ciudadano, omitiendo barreras como las actuales de depender de la voluntad de un ente político o de subsumir a los servidores públicos de separarse con 
antelación a la mitad de su encargo para poder contender en la siguiente elección por partido diverso o como candidatos independientes.

\section{Bibliografía:}

Báez Villaseñor, María Estela. Un largo camino: la lucha por el sufragio femenino en Estados Unidos. México: Scielo, 2010.

Baldez, Lisa. "Cuotas versus primarias: La nominación de candidaturas mujeres en México". En Mujer y Política: Impacto de las cuotas de género en Améerica Latina (2012): 157-77.

Bobbio, Nolberto. Introduziones aT. Hobbs, Opera Politiche. Turín: Utee, 1959.

Calderon Hernández, Carlos Alfonso. "La reelección presidencial en latinoamerciana: una avance en el caudillismo y un retroceso en la democracia". Derechos y Realidad, n² 24, II sementre (2014): 128-30.

Facio, Alda. El derecho a la igualdad de mujeres y hombres. Interpretación de los principios de igualdad y no discriminación para los derechos humanos de las mujeres en los instrumentos del Sistema Interamericano. San José, Costa Rica: IIDH, 2009.

Gutiérrez S., Raúl. Los elementos del conocimiento en general. México: Esfinge, 2000.

González Casanova, Pablo. La Democracia en México. México: Era, 2008. Jaimes Delgado, David. La Reelección Legislativa inmediata una propuesta para fortalecer el Poder Legislativo en México. México: Porrúa, 2011.

Lassalle, Ferdinand. ¿Qué es una Constitución?. Berlín: Ariel DERECHO, 2012.

Nohlen, Dieter, "La reelección", cap. XV, en Tratado de Derecho Electoral Comparado de América Latina. México: Fondo de Cultura Económica, Instituto Interamericano de Derechos Humanos, Universidad de Heidelberg, International IDEA, Tribunal Electoral del Poder Judicial de la Federación, Instituto Federal Electoral, 2007.

Sánchez Medero, Gema. Los Partidos Políticos: Organización y Funcionamiento. Madrid: Trotta, 2012. 
Spagna Musso, Enrico. "La Tutela Constitucional de los intereses colectivos en el Estado de Democracia Pluralista". Estudios Políticos del Centro de Estudios Constitucionales de Madrid, $n^{\circ} 4$ (1978): 57-66.

Tuñón, Esperanza. "También somos protagonistas de la historia de México", en Cuadernos para la Mujer, $n^{\circ}$ 5. México: EMAS,1987, 5-6.

Wróblewski, Jerzy. Sentido y hecho en el derecho. México: Fontamara, 2008.

\section{Webgrafia:}

Carta Democrática Interamericana, OEA, Lima Perú, 11 de septiembre de 2001 , disponible

en: www.ordenjuridico.gob.mx/TratInt/Derechos\%20Humanos/OTROS\%2000.pd f

CPEUM. Ver Constitución Política de los Estados Unidos Mexicanos, disponible

en:

http://www.congresoson.gob.mx:81/Content/Doc_leyes/doc_446.pdf

Convención sobre los derechos políticos de la Mujer, Nueva York, 31 de marzo de 1953, disponible en: www.ordenjuridico.gob.mx/TratInt/Derechos\%20Humanos/D45.pdf Decreto, por el que se reforman, adicionan y derogan diversas disposiciones de la Constitución Política, en materia política-electoral (Diario Oficial de la Federación, 10 febrero de 2014), consultable en:http://www.dof.gob.mx/nota_detalle.php?codigo=5332025\&fecha=10/02/2 014

Iniciativa presentada por la Senadora Mariana Gómez del Campo Gurza, con proyecto de decreto por el que se reforman los artículos 59, el segundo párrafo de la fracción II del artículo 116 y el segundo párrafo de la fracción I base primera del artículo 122 de la Constitución Política de los Estados Unidos Mexicanos. (Cámara de Senadores, 9 octubre de 2012, publicado en el Diario Oficial de la Federación el 10 de febrero de 2014, en el Decreto por el que se reforman, adicionan y derogan diversas disposiciones de la 
Constitución Política de los Estados Unidos Mexicanos, en materia políticaelectoral) 49-50,

consultable

en

http://www.diputados.gob.mx/sedia/biblio/prog_leg/135_DOF_10feb14.pdf Jurisprudencia 6/2015. PARIDAD DE GÉNERO. DEBE OBSERVARSE EN LA POSTULACIÓN DE CANDIDATURAS PARA LA INTEGRACIÓN DE ÓRGANOS DE REPRESENTACIÓN POPULAR FEDERALES, ESTATALES Y MUNICIPALES, consultable

en:

http://sief.te.gob.mx/iuse/tesisjur.aspx?idtesis=6/2015\&tpoBusqueda=S\&sWo $r d=6 / 2015$

Jurisprudencia 43/2014. ACCIONES AFIRMATIVAS. TIENEN SUSTENTO EN EL PRINCIPIO CONSTITUCIONAL Y CONVENCIONAL DE IGUALDAD MATERIAL, consultable

en:

http://sief.te.gob.mx/iuse/tesisjur.aspx?idtesis=43/2014\&tpoBusqueda=S\&sW ord $=43 / 2014$

Molina Brizuela, Yanko. "Teoría de Género en Contribuciones a las Ciencias Sociales". Eumed.Net Enciclopedia

Virtual (2010):

http://www.eumed.net/rev/cccss/10/ymb2.htm

Sentencia SUP-RAP-134/2015, ( Tribunal Electoral del Poder Judicial de la Federación, 6 de mayo de 2015), consultable en: http://portal.te.gob.mx/colecciones/sentencias/html/SUP/2015/RAP/SUPRAP-00134-2015.htm

Sentencia SUP-RAP- 825/2016, (Tribunal Electoral del Poder Judicial de la Federación 21 de diciembre de 2016), consultable en: http://portal.te.gob.mx/colecciones/sentencias/html/SUP/2016/REC/SUP. REC-00825-2016.htm

\section{Normatividad:}

Tesis-2003350 I.40.A.9. PRINCIPIOS DE UNIVERSALIDAD, INTERDEPENDENCIA, INDIVISIBILIDAD Y PROGRESIVIDAD DE LOS DERECHOS HUMANOS. EN QUÉ CONSISTEN. (Abril de 2013, Libro XIX, Tomo 3, Semanario Judicial de la Federación y su Gaceta), 2254. 